\title{
Rehabilitation guidance for hip fracture patients during the COVID-19 pandemic using chat software: A new model
}

\author{
Shuai-Shuai Gao ${ }^{\mathrm{a}, \mathrm{b}}$, Yan-Jun Wang ${ }^{\mathrm{a}}$, Guo-Xun Zhang ${ }^{\mathrm{b}}$ and Wen-Ting Zhang ${ }^{\mathrm{b}, *}$ \\ ${ }^{a}$ Department of Traumatology and Orthopedic Surgery, Xi'an Daxing Hospital, Shaanxi, China \\ ${ }^{\mathrm{b}}$ International Doctoral School, University of Seville, Seville, Spain
}

Received 28 October 2020

Accepted 25 March 2021

\begin{abstract}
.
BACKGROUND: Hip fractures are serious fractures for the elderly. The rehabilitation of patients with hip fractures has been greatly affected by the coronavirus disease 2019 (COVID-19) pandemic.

OBJECTIVE: We have piloted a new model for tracking patients and providing rehabilitation guidance that uses WeChat. The purpose of this study is to explore the role of chat software in rehabilitation guidance for hip fracture patients during COVID-19. METHODS: Patients treated for hip fractures from February 1 to April 30, 2020 were randomly divided into a control group and an observation group. The control group was given conventional discharge guidance, while the observation group also followed up the patients using WeChat to guide the exercise. Satisfaction, the Harris Hip Score, complications and the mortality of the two groups after discharge were compared.

RESULTS: The incidence of complications and mortality in the observation group were significantly lower than in the control group: $p=0.022$ and $p=0.048$, respectively. The Harris Hip Score and satisfaction were significantly better than the control group's: $p=0.000$ and $p=0.007$, respectively.

CONCLUSION: During the COVID-19 pandemic, it is very helpful to use WeChat software or other social software with similar functions (such as WhatsApp and Facebook) to guide the rehabilitation of hip fractures.
\end{abstract}

Keywords: COVID-19, hip fracture, rehabilitation, mortality

\section{Introduction}

The novel coronavirus pneumonia is an acute infectious pneumonia [1]. On February 11, 2020, the World Health Organization (WHO) named it coronavirus disease 2019 (COVID-19). Since its outbreak in Wuhan, it has infected more than 35 million people and led to $1,000,000$ deaths worldwide [2], causing serious damage to people's daily life and health, especially that of

\footnotetext{
${ }^{*}$ Corresponding author: Wen-Ting Zhang, International Doctoral School, University of Seville, Plaza Comunidad Castilla la Mancha, No. 6, Seville, 41008, Spain. Tel.: +34 655546227; E-mail: 724521882@qq.com.
}

the elderly. Because of their increased comorbidities and poor resistance, the mortality rate is significantly higher than that of other groups [3].

Hip fractures are serious fractures that often occur in the elderly. With the advent of global aging, it is estimated that by 2050, the occurrence of hip fractures will increase to 6.5 million annually [4]. The COVID-19 pandemic has also had a great impact on hip fractures. A study from Spain [5] showed that during the pandemic, the incidence of hip fractures had significantly reduced compared with the same period in previous years, and the length of hospital stay had also significantly shortened. However, Catellani [6] and Mi [7] reported that the 30-day overall mortality rate of hip fractures in- 


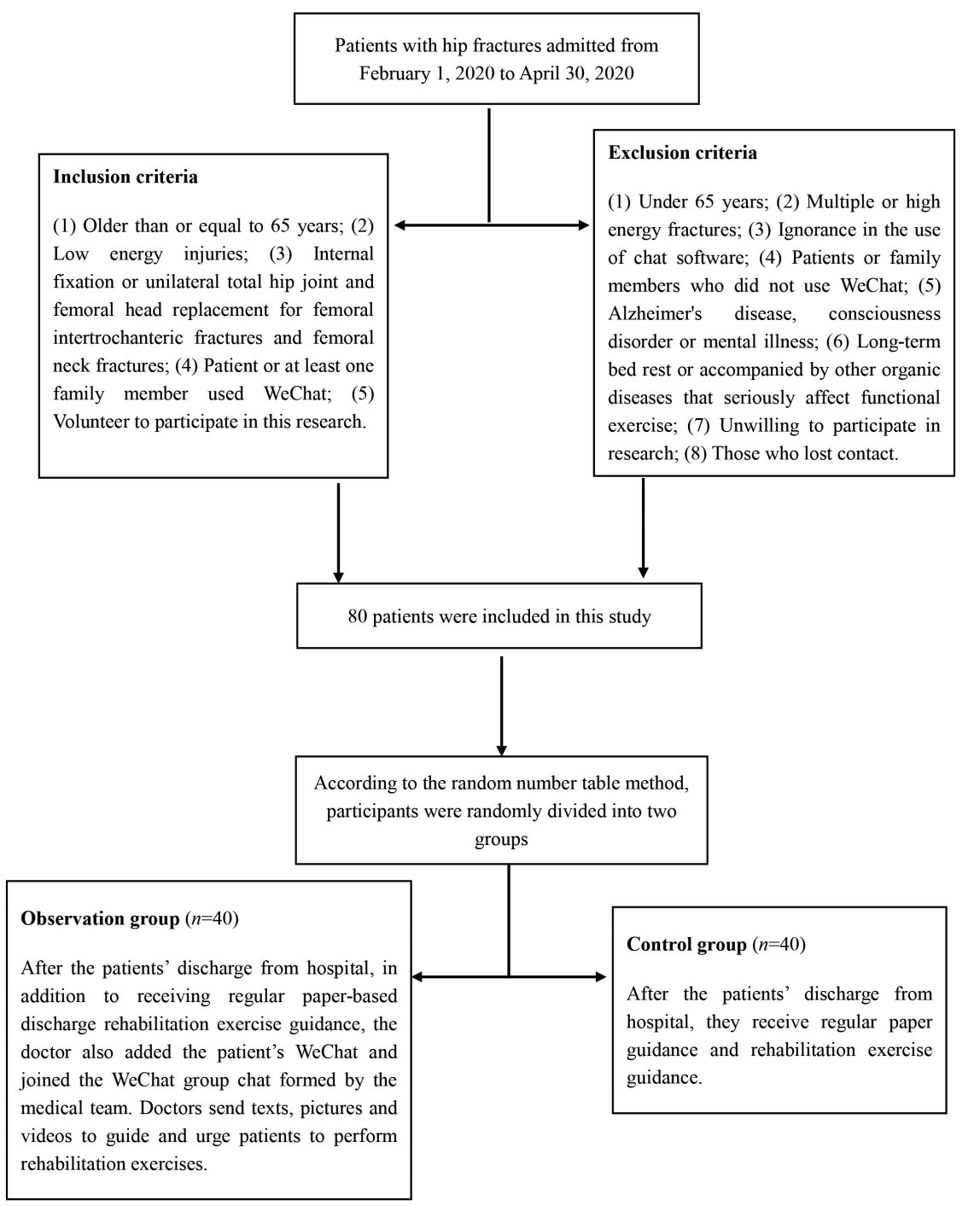

Fig. 1. Flowchart of patient recruitment, randomization and testing.

creased significantly compared with the same period in previous years. Due to the government's imposition of martial law, non-emergency departments in many hospitals in Wuhan have been ordered to close or have been requisitioned for treating COVID-19 patients, such as rehabilitation departments [8]. This has made it difficult for patients with hip fractures to receive effective rehabilitation guidance after their hurriedly discharge from hospital, leading to the weakened effect of treatment effects and/or poor quality of life [9].

With advances in science and technology, smart phone chat software (such as WhatsApp, Facebook and WeChat) has become an indispensable communication tool for everyone [10]. Because of its high popularity, speed, convenience, and ease of operation, it has been applied in cancer prevention and management [11,12]. This article will report on the effect of our department's use of WeChat on the rehabilitation guidance of hip fracture patients during the COVID-19 pandemic, hoping to add to the ideas and methods for the rehabilitation guidance of hip fractures during the pandemic.

\section{Materials and methods}

\subsection{Patients}

The flowchart of patient recruitment, randomization and testing is shown in Fig. 1. The patients with hip fracture who were treated in trauma and orthopedic 
surgery from February 1, 2020 to April 30, 2020 were randomly divided into a control and an observation group according to the random number table method. The following variables were studied in each patient: gender, age, classification of the American Society of Anesthesiologists (ASA, Grades 1 and 2 were low risk, while Grades 3 and 4 were high risk), number of comorbidities (divided into two or less and three or more), length of hospital stay, and timing of surgery (early and late). This study was approved by the Xi'an Daxing Hospital Ethics Committee and all patients signed an informed consent form prior to the study.

\subsection{Inclusion and exclusion criteria}

Inclusion criteria: (1) Older than or equal to 65 years; (2) Low energy injuries; (3) Internal fixation or unilateral total hip joint and femoral head replacement for femoral intertrochanteric fractures and femoral neck fractures; (4) Patient or at least one family member used WeChat; (5) Volunteer to participate in this research.

Exclusion criteria: (1) Under 65 years; (2) Multiple or high energy fractures; (3) Ignorance of the use of chat software; (4) Patients or family members who did not use WeChat; (5) Alzheimer's disease, consciousness disorder or mental illness; (6) Long-term bed rest or accompanied by other organic diseases that seriously affect functional exercise; (7) Unwilling to participate in research; (8) Those who lost contact.

\subsection{Methods}

Specific operation: When control group patients were discharged from the hospital, they were given the usual paper discharge instructions and rehabilitation exercise guidance. In addition to these measures for the observation group, the doctors also added the patients' WeChat and joined the WeChat group chat formed by the medical team. Doctors transmitted text, pictures, voice and video to the group to guide and urge patients to perform rehabilitation exercises, such as correct sitting posture, when to use abduction, and when to abandon it; patients could also consult their condition and upload their own rehabilitation results through WeChat. For special patients, doctors would provide personalized and targeted guidance, for example, patients who were used to putting the affected limb on the other leg to prevent the prosthesis from coming out were send pictures or videos of correct posture and prohibited actions through WeChat.

\subsection{Observation indicators}

Observation indicators: The following data was ob- tained through questionnaire surveys of telephone and chat groups. (a) Mortality and complications within 60 days of discharge. (b) Harris Hip Score at discharge and 60 days after discharge. (c) Patient satisfaction (good or bad).

\subsection{Statistical analysis}

Data analysis was performed using SPSS statistical software (SPSS, version 17.0). The parametric distributions were compared by means of the sample distribution of means with the Student's $t$-test, while the qualitative variables were compared with the Chi-square, with a significance level of $p<0.05$.

\section{Results}

A total of 80 patients with hip fractures were enrolled, including 30 males and 50 females. The control group and the observation group each had 40 cases. The clinical data of the two groups of patients before discharge is shown in Table 1 . There was no difference between the two groups which were comparable. Perhaps due to the small prevalence of COVID-19 in our province, all patients tested negative for the virus.

Comparison of complications, mortality, Harris Hip Score and satisfaction between the two groups after discharge. As shown in Table 2, 60 days after discharge, there were six deaths in the control group, and one in the observation group; 15 patients in the control group had complications, while only six did in the observation group. The main complications were deep vein thrombosis, urinary tract infection, heart failure, incision infection, lung infection, dislocation of the prosthesis, and fracture around the prosthesis. The incidence of complications and mortality in the observation group were significantly lower than that of the control group: $p=0.022$ and $p=0.048$, respectively. The Harris Hip Score and satisfaction were significantly better than that of the control group: $p=0.000$ and $p=0.007$, respectively.

\section{Discussion}

Due to the impact of the COVID-19 pandemic and the national martial law policy, hip fracture patients and their families were more willing to leave hospital earlier [13] and were also afraid of going out to recover. In addition, the reduction of treatment activities in the 
Table 1

Comparison of the clinical data of the two groups of patients before discharge

\begin{tabular}{|c|c|c|c|c|}
\hline & Control group $(n=40)$ & Observation group $(n=40)$ & t or $X^{2}$ & $P$-value \\
\hline Age (years, $x \pm s$ ) & $77.83 \pm 5.92$ & $78.13 \pm 5.58$ & 0.233 & 0.816 \\
\hline \multicolumn{5}{|l|}{ Sex } \\
\hline Male & 14 & 16 & 0.213 & 0.644 \\
\hline Female & 26 & 24 & & \\
\hline \multicolumn{5}{|l|}{ Number of comorbidities } \\
\hline$<3$ & 17 & 15 & 0.208 & 0.648 \\
\hline$\geqslant 3$ & 23 & 25 & & \\
\hline \multicolumn{5}{|l|}{ ASA classification } \\
\hline Low risk & 17 & 16 & 0.052 & 0.820 \\
\hline High risk & 23 & 24 & & \\
\hline \multicolumn{5}{|l|}{ Timing of surgery } \\
\hline Early ( $<48$ horas) & 17 & 13 & 0.853 & 0.356 \\
\hline Delay ( $\geqslant 48$ horas) & 23 & 27 & & \\
\hline Complications & $3(7.5 \%)$ & $2(5 \%)$ & 0.213 & 0.644 \\
\hline Deep vein thrombosis & 1 & 1 & & \\
\hline Urinary tract infection & 1 & & & \\
\hline Heart failure & & 1 & & \\
\hline Postoperative delirium & 1 & & & \\
\hline Harris Hip Score at discharge & $59.60 \pm 4.6$ & $58.80 \pm 5.2$ & 0.730 & 0.468 \\
\hline Hospital stay (days, $\mathrm{x} \pm \mathrm{s}$ ) & $7.53 \pm 2.43$ & $7.70 \pm 2.49$ & 0.318 & 0.751 \\
\hline
\end{tabular}

Table 2

Comparison of complications, mortality, hip score and satisfaction between the two groups after discharge

\begin{tabular}{|c|c|c|c|c|}
\hline & Control group $(n=40)$ & Observation group $(n=40)$ & $\mathrm{t}$ or $\mathrm{X}^{2}$ & $P$-value \\
\hline Complications & $15(37.5 \%)$ & $6(15 \%)$ & 5.230 & 0.022 \\
\hline Deep vein thrombosis & 4 & 1 & & \\
\hline Urinary tract infection & 3 & 1 & & \\
\hline Heart failure & 3 & 2 & & \\
\hline Incision infection & 1 & 0 & & \\
\hline Lung infection & 3 & 0 & & \\
\hline Dislocation of the prosthesis & 1 & 0 & & \\
\hline Fracture around the prosthesis & 0 & 1 & & \\
\hline Mortality & $6(15 \%)$ & $1(2.4 \%)$ & 4.006 & 0.048 \\
\hline \multicolumn{5}{|l|}{ Satisfaction } \\
\hline Good & 31 & 39 & 7.314 & 0.007 \\
\hline Bad & 9 & 1 & & \\
\hline Harris score 60 days after discharge & $81.33 \pm 7.4$ & $88.28 \pm 5.5$ & 4.776 & 0.000 \\
\hline
\end{tabular}

rehabilitation department or clinic has significantly reduced the rehabilitation exercise of patients, leading to a worse prognosis for patients [14]. We therefore tried a new model: using WeChat to follow up patients and provide rehabilitation guidance. As far as we know, this is the first study to propose and report the effect of using chat software on hip fractures during the COVID-19 pandemic, and it obtained practical and promotional results.

During the COVID-19 pandemic, patients with hip fractures suffered from increased malnutrition [15], reduced activities, lacked of effective rehabilitation guidance and other factors, resulting in a significant increase in postoperative complications and overall mortality $[16,17]$. By sing chat software, it was possible to guide and urge patients to exercise, answer their doubts, and reduce the pressure of patients with medical diffi- culties, thereby reducing the complications and 60-day mortality rate of patients after surgery.

Hip fractures have a high disability rate, and less than half of the people can regain their pre-injury activities [18], especially when patients do not receive effective rehabilitation guidance. The Harris Hip Score is a measure of dysfunction: the higher the score, the better the outcome for the individual. The maximum possible score is 100 , so results can be interpreted thus [19]: $<70=$ poor result; $70-80=$ fair, $80-90=$ good, and 90-100 = excellent. By using chat software, uploading rehabilitation videos, and urging patients to exercise correctly, the patient's activity ability could be significantly improved. Sixty days after discharge, the Harris Hip Score was significantly higher than that of the control group. 
Due to the current pandemic, the work of doctors was intense and heavy and the treatment process of patients after admission had become complicated and slow [5,20]. In addition, communication between doctors and patients had decreased with the shortening of hospital stays. This increased the dissatisfaction of patients with medical treatment. However, through the convenience of chat software, patients and doctors were closely connected, the treatment process to be continued without interruption, significantly improving patient satisfaction, and better coordinating doctors' rehabilitation guidance. This was consistent with Narasimha's research results [21].

Although the results of this article were satisfactory, it has the following shortcomings. Firstly, the sample size was small, a larger sample is needed for research. Secondly, the cases included were all COVID19 negative, so the result in COVID-19 positive cases is not known. Thirdly, the follow-up time was short and the long-term effects remain to be studied. Finally, the COVID-19 pandemic is not over and its impact on hip fractures is still changing.

\section{Conclusion}

During the COVID-19 pandemic, the use of WeChat software or other social software with similar functions (such as WhatsApp and Facebook) to provide rehabilitation guidance for hip fractures reduced patient mortality, optimized patient mobility, and improved patient satisfaction. It is worth promoting this novel idea and model.

\section{Conflict of interest}

The authors have no conflicts of interest to declare.

\section{Funding}

The author(s) received no financial support for the research, authorship, and/or publication of this article.

\section{References}

[1] Li Y, Li J, Zhang Y, Dai L, Li L, Liu J, et al. Development of an automatic integrated gene detection system for novel severe acute respiratory syndrome-related coronavirus (SARSCoV2). Emerging Microbes \& Infections. 2020; 9(1): 1489-96.
[2] https://www.who.int/emergencies/diseases/novel-coronavirus -2019. Last visit as of October 4, 2020.

[3] Islam N, Khunti K, Dambha-Miller H, Kawachi I, Marmot M. COVID-19 mortality: A complex interplay of sex, gender, and ethnicity. European journal of public health. 2020.

[4] Cooper C, Cole ZA, Holroyd CR, Earl SC, Harvey NC, Dennison EM, et al. Secular trends in the incidence of hip and other osteoporotic fractures. Osteoporosis International: A Journal Established as Result of Cooperation Between the European Foundation for Osteoporosis and the National Osteoporosis Foundation of the USA. 2011; 22(5): 1277-88.

[5] Minarro JC, Zamorano-Moyano C, Urbano-Luque MT, Arenas-de Larriva AP, Izquierdo-Fernández A, QuevedoReinoso R. Is COVID-19 affecting the incidence of hip fractures? Injury. 2020.

[6] Catellani F, Coscione A, D’ Ambrosi R, Usai L, Roscitano C, Fiorentino G. Treatment of proximal femoral fragility fractures in patients with COVID-19 during the SARS-CoV-2 outbreak in northern Italy. The Journal of Bone and Joint Surgery American Volume. 2020; 102(12): e58.

[7] Mi B, Chen L, Xiong Y, Xue H, Zhou W, Liu G. Characteristics and early prognosis of COVID-19 infection in fracture patients. The Journal of Bone and Joint Surgery American Volume. 2020; 102(9): 750-8.

[8] Bartolo M, Intiso D, Lentino C, Sandrini G, Paolucci S Zampolini M. Urgent measures for the containment of the coronavirus (Covid-19) epidemic in the neurorehabilitation/rehabilitation departments in the phase of maximum expansion of the epidemic. Frontiers in Neurology. 2020; 11 : 423.

[9] Parnes N, Tousant C, Perrine J, DeFranco MJ. Outpatient orthopedic rehabilitation in new york state during the COVID-19 pandemic: therapist perspectives. Orthopedics. 2020: 1-3.

[10] https://www.inc.com/larry-kim/the-top-7-messenger-apps-inworld.html.

[11] Park S, Kim JY, Lee JC, Kim HR, Song S, Kwon H, et al. Mobile phone app-based pulmonary rehabilitation for chemotherapy-treated patients with advanced lung cancer: pilot study. JMIR mHealth and uHealth. 2019; 7(2): e11094.

[12] Zheng C, Chen X, Weng L, Guo L, Xu H, Lin M, et al. Benefits of mobile apps for cancer pain management: systematic review. JMIR mHealth and uHealth. 2020; 8(1): e17055.

[13] Bellan M, Gavelli F, Hayden E, Patrucco F, Soddu D, Pedrinelli AR, et al. Pattern of emergency department referral during the Covid-19 outbreak in Italy. Panminerva medica. 2020.

[14] Xu BY, Yan S, Low LL, Vasanwala FF, Low SG. Predictors of poor functional outcomes and mortality in patients with hip fracture: a systematic review. BMC Musculoskeletal Disorders. 2019; 20(1): 568 .

[15] Bencivenga L, Rengo G, Varricchi G. Elderly at time of COronaVIrus disease 2019 (COVID-19): possible role of immunosenescence and malnutrition. GeroScience. 2020; 42(4): 108992.

[16] LeBrun DG, Konnaris MA, Ghahramani GC, Premkumar A, DeFrancesco CJ, Gruskay JA, et al. Hip fracture outcomes during the COVID-19 pandemic: early results from new york. Journal of Orthopaedic Trauma. 2020; 34(8): 403-10.

[17] Egol KA, Konda SR, Bird ML, Dedhia N, Landes EK, Ranson RA, et al. Increased mortality and major complications in hip fracture care during the COVID-19 pandemic: a new york city perspective. Journal of Orthopaedic Trauma. 2020; 34(8): 395-402. 
[18] Dyer SM, Crotty M, Fairhall N, Magaziner J, Beaupre LA, Cameron ID, et al. A critical review of the long-term disability outcomes following hip fracture. BMC Geriatr. 2016; 16(1): 158.

[19] Harris WH. Traumatic arthritis of the hip after dislocation and acetabular fractures: treatment by mold arthroplasty. An end-result study using a new method of result evaluation. The Journal of Bone and Joint Surgery American Volume. 1969; 51(4): 737-55.
[20] Katayama Y, Kiyohara K, Kitamura T, Hayashida S, Shimazu T. Influence of the COVID-19 pandemic on an emergency medical service system: a population-based, descriptive study in Osaka, Japan. Acute Medicine \& Surgery. 2020; 7(1): e534.

[21] Narasimha S, Agnisarman S, Chalil Madathil K, Gramopadhye A, McElligott JT. Designing home-based telemedicine systems for the geriatric population: an empirical study. Telemedicine Journal and E-health: The Official Journal of the American Telemedicine Association. 2018; 24(2): 94-110. 